\title{
Assessing exercise cardiac reserve using real-time cardiovascular magnetic resonance
}

Thu-Thao Le ${ }^{1 *}$, Jennifer Ann Bryant ${ }^{1}$, Alicia Er Ting ${ }^{1}$, Pei Yi Ho ${ }^{1}$, Boyang Su${ }^{1}$, Raymond Choon Chye Teo ${ }^{2}$, Julian Siong-Jin Gan ${ }^{3}$, Yiu-Cho Chung ${ }^{3}$, Declan P. O'Regan ${ }^{4}$, Stuart A. Cook ${ }^{1,5,6+}$ and Calvin Woon-Loong Chin ${ }^{1,5+}$

\begin{abstract}
Background: Exercise cardiovascular magnetic resonance (ExCMR) has great potential for clinical use but its development has been limited by a lack of compatible equipment and robust real-time imaging techniques. We developed an exCMR protocol using an in-scanner cycle ergometer and assessed its performance in differentiating athletes from non-athletes.

Methods: Free-breathing real-time CMR (1.5T Aera, Siemens) was performed in 11 athletes (5 males; median age 29 [IQR: 28-39] years) and 16 age- and sex-matched healthy volunteers (7 males; median age 26 [interquartile range (IQR): 25-33] years). All participants underwent an in-scanner exercise protocol on a CMR compatible cycle ergometer (Lode BV, the Netherlands), with an initial workload of $25 \mathrm{~W}$ followed by $25 \mathrm{~W}$-increment every minute. In 20 individuals, exercise capacity was also evaluated by cardiopulmonary exercise test (CPET). Scan-rescan reproducibility was assessed in 10 individuals, at least 7 days apart.

Results: The exCMR protocol demonstrated excellent scan-rescan (cardiac index (Cl): $0.2 \pm 0.5 \mathrm{~L} / \mathrm{min} / \mathrm{m}^{2}$ ) and inter-observer (ventricular volumes: $1.2 \pm 5.3 \mathrm{~mL}$ ) reproducibility. Cl derived from exCMR and CPET had excellent correlation $(r=0.83, p<0.001)$ and agreement $\left(1.7 \pm 1.8 \mathrm{~L} / \mathrm{min} / \mathrm{m}^{2}\right)$. Despite similar values at rest $(P=0.87)$, athletes had increased exercise $\mathrm{Cl}$ compared to healthy individuals (at peak exercise: 12.2 [IQR: 10.2-13.5] L/min $/ \mathrm{m}^{2}$ versus 8.9 [IQR: $\left.7.5-10.1\right] \mathrm{L} / \mathrm{min} / \mathrm{m}^{2}$, respectively; $P<0.001$ ). Peak exercise $\mathrm{Cl}$, where image acquisition lasted 13-17 s, outperformed that at rest (c-statistics $=0.95$ [95\% confidence interval: 0.87-1.00] versus 0.48 [95\% confidence interval: $0.23-0.72$ ], respectively; $P<0.0001$ for comparison) in differentiating athletes from healthy volunteers; and had similar performance as $\mathrm{VO}_{2 \max }$ (c-statistics $=0.84$ [95\% confidence interval $=$ 0.62-1.00]; $P=0.29$ for comparison).
\end{abstract}

Conclusions: We have developed a novel in-scanner exCMR protocol using real-time CMR that is highly reproducible. It may now be developed for clinical use for physiological studies of the heart and circulation.

Keywords: Cardiovascular magnetic resonance, Supine bike ergometer, Exercise physiology, Cardiopulmonary exercise test

\footnotetext{
* Correspondence: le.thu.thao@nhcs.com.sg

${ }^{\dagger}$ Equal contributors

${ }^{1}$ National Heart Centre Singapore, 5 Hospital Drive, Singapore 169609,

Singapore

Full list of author information is available at the end of the article
} International License (http://creativecommons.org/licenses/by/4.0/), which permits unrestricted use, distribution, and reproduction in any medium, provided you give appropriate credit to the original author(s) and the source, provide a link to the Creative Commons license, and indicate if changes were made. The Creative Commons Public Domain Dedication waiver (http://creativecommons.org/publicdomain/zero/1.0/) applies to the data made available in this article, unless otherwise stated. 


\section{Background}

Cardiac exercise testing is commonly used to detect underlying cardiovascular abnormalities that are not apparent at rest. Cardiovascular magnetic resonance (CMR) provides accurate assessment of cardiac volumes and function with excellent reproducibility compared to other standard imaging modalities $[1,2]$ but its application in stress testing has been limited to pharmacological agents.

Up until recently, the lack of suitable CMR-compatible exercise equipment and real-time imaging techniques have precluded accurate cardiac assessment of exercise physiology. Early studies in exercise CMR (exCMR) were performed using either breath-hold procedures that are not physiological or long free-breathing image acquisitions [3-5]. Although improvements in CMR technology have shortened the duration of exCMR imaging, these studies used a CMR-compatible treadmill [6-8]. The strengths of using treadmill exCMR are its validated diagnostic [9] and prognostic value and the ability to perform 12-lead electrocardiogram during exercise stress (the Duke score that also carries prognostic value) [10, 11]. However, a major limitation of performing treadmill exCMR is the obvious time delay needed to transfer the patient from the treadmill into the scanner.

In contrast, a supine cycle ergometer attached to the scan table will allow patients to exercise while in the bore. There are many advantages for exCMR particularly as images are acquired during the intermediate stages as well as at peak exercise, thus providing a large added value for statistical analyses of quantitative indices (e.g. stroke volume) and for repeated appreciation of qualitative changes (e.g. wall motion). However, excessive motion during exercise poses a challenge in image acquisition. Ungated real-time cine imaging and retrospective synchronisation of respiratory cycles have been used [5]. However, this translated to increased image acquisition time and complex image post-processing. We propose an approach of acquiring cine images at every stage of exercise during a brief period of exercise cessation to reduce artefacts from excessive motion and ECG-gating.

Using a CMR-compatible cycle ergometer and realtime CMR, we aimed to evaluate the feasibility and reproducibility of our exercise protocol; and to examine its potential to differentiate athletes from healthy volunteers.

\section{Methods}

\section{Study population}

A total of 11 athletes and 16 age- and sex-matched healthy volunteers were recruited to the study. Healthy volunteers did not have any diagnosed cardiac conditions and cardiovascular risk factors (hypertension, diabetes mellitus and hyperlipidemia). All the athletes competed in national/international events and trained more than $10 \mathrm{~h}$ a week in a variety of sports: triathlons $(n=7)$, long distance running $(n=1)$, rowing $(n=1)$, rugby $(n=1)$ and badminton $(n=1)$. We used the wellvalidated General Practice Physical Activity Questionnaire to assess physical activity levels in all participants, and classified them into four categories: inactive, moderately inactive, moderately active and active [12]. A fivepoint score was used to assess participant's experience during exCMR ( 1 = would not do it again to 5 = highly satisfied; 3 = neutral).

The study was conducted in accordance with the Declaration of Helsinki and approved by the Singhealth Centralised Institutional Review Board. Written informed consent was obtained from all individuals.

\section{Exercise CMR protocol}

Exercise was performed using a programmable supine ergometer (Lode BV, Netherlands) fitted onto the CMR scanner table (1.5T MAGNETOM Aera, Siemens, Erlangen, Germany) (Figs. 1 and 2). Images were acquired using the 60-channel cardiac coils (30 anterior and 30 posterior elements).

After obtaining the baseline images, the participants were asked to cycle at an initial workload of $25 \mathrm{~W}$, with cadence maintained at least $70 \mathrm{rpm}$ for $1 \mathrm{~min}$. Workload was increased by $25 \mathrm{~W}$ every minute until exhaustion. Free-breathing imaging was performed at the end of every stage during a brief period of stopping exercise. This is to avoid poor ECG signal and excessive motion artefacts that might result during exercise. Blood pressure and heart rate were recorded at every stage of exercise.

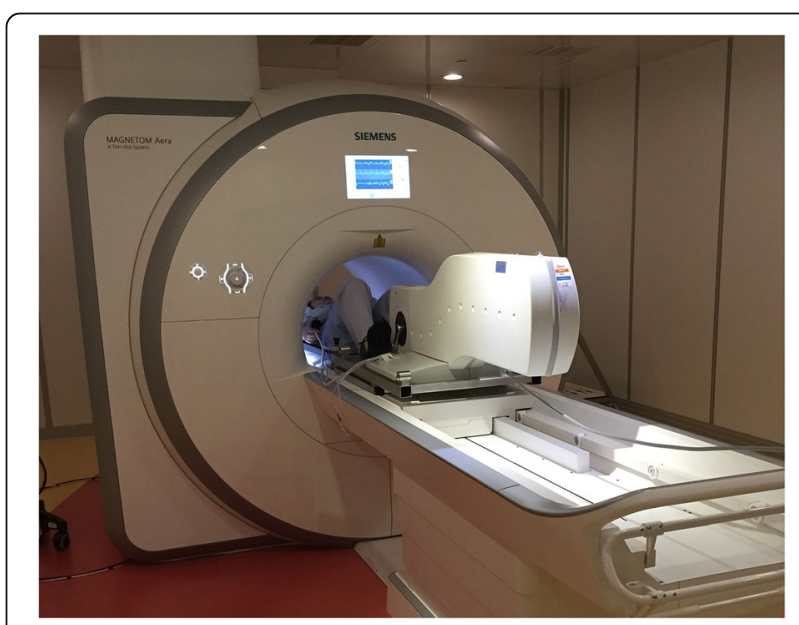

Fig. 1 Set up of the cycle ergometer in CMR scanner 


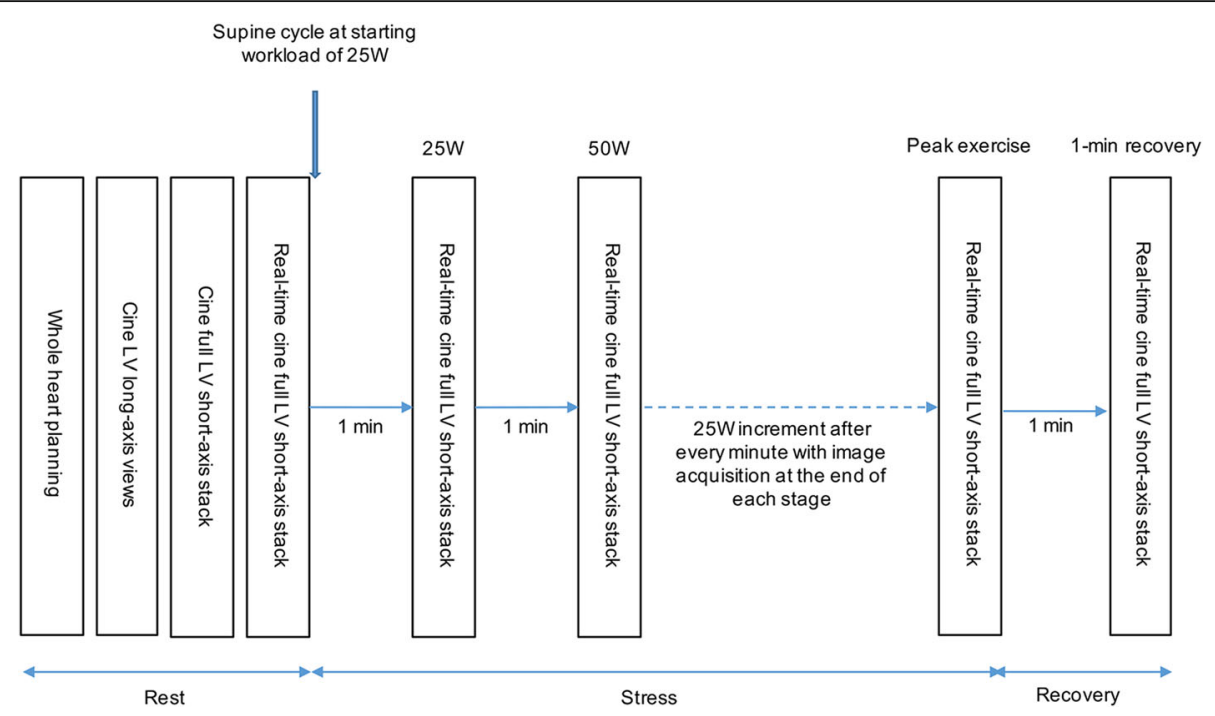

Fig. 2 Exercise CMR imaging protocol

The imaging sequences and parameters were as follows:

Balanced steady-state free precession (bSSFP): standard long axis (vertical, horizontal long axis, sagittal LV outflow tract) and short axis cines (extending from the base to apex) were performed in all patients before initiating exercise $(8 \mathrm{~mm}$ thick and $2 \mathrm{~mm}$ gap; TE $1.2 \mathrm{~ms}$; TR $3 \mathrm{~ms} ; 280-320 \mathrm{~mm}$ field of view; 13 segments per phase; acquired matrix size $205 \times 256$ pixels; acceleration factor of 2 ; acquired voxel size $1.6 \times 1.3 \times 8.0 \mathrm{~mm} ; 30$ phases per cardiac cycle).

Real-time bSSFP short axis cines: prospective ECG-gated free-breathing image acquisition of 10-13 short axis cine slices, extending from the base to apex was performed at each stage of the exercise $(8 \mathrm{~mm}$ thick and $2 \mathrm{~mm}$ gap; TE $0.99 \mathrm{~ms}$; TR $2.3 \mathrm{~ms} ; 225 \times 300 \mathrm{~mm}$ field of view; phase FOV 75\%; acquired matrix size $68 \times 128$ pixels; phase resolution $71 \%$; acceleration factor of 4 ; acquired voxel size $3.3 \times 2.3 \times 8.0 \mathrm{~mm}$ ), temporal resolution $39.1 \mathrm{~ms}$. Two different acquisition duration per slice were used in image acquisition: 1500 $\mathrm{ms}$ for heart rate less than $80 \mathrm{bpm}$ (scan duration between 17 and $22 \mathrm{~s}$ ); and $1200 \mathrm{~ms}$ for heart rate greater than $80 \mathrm{bpm}$ (scan duration between 13 and $17 \mathrm{~s}$ ). The rationale of using different acquisition durations was to obtain sufficient frames per cardiac cycle to capture end-diastole and end-systole at the different exercise stages. Using this approach, we were able to capture at least 2 cardiac cycles per exercise stage (Additional file 1: Video S1, Additional file 2: Video S2 and Additional files 3: Video S3).

\section{Image analysis}

Left ventricular (LV) endocardial borders in short axis cine images, at end diastole and end systole, were manually contoured at baseline and at each exercise stage (CVI42, Circle Cardiovascular Imaging Inc., Canada). Stroke volume (SV) was measured as the difference between maximum volume (end-diastolic volume, EDV) and minimum volume (end-systolic volume, ESV) across the cardiac cycles (Fig. 3). Additional steps to manually select and compose the end-diastolic and end-systolic phases may be required if there was misalignment of phases due to ECG mistriggering. Cardiac output (CO) was calculated as: $\mathrm{CO}=\mathrm{SV} \times \mathrm{HR}$. All measured volumes and cardiac output were indexed to body surface area (DuBois formula).

\section{Reproducibility and validation}

In 10 individuals, a repeat scan using the same exercise protocol was performed at least 7 days from the first scan to assess scan-rescan reproducibility.

A total of 20 individuals (healthy volunteers, $n=10$ and athletes, $n=10$ ) underwent additional cardiopulmonary exercise test (CPET), the gold standard of assessing exercise capacity. CPET was performed on an upright cycle ergometer (Vmax Encore E229C, USA) with a similar incremental protocol beginning at $50 \mathrm{~W}$ and $25 \mathrm{~W}$ increments every minute until exhaustion. Oxygen consumption at baseline $\left(\mathrm{VO}_{2}\right)$ and at peak exercise $\left(\mathrm{VO}_{2 \max }\right)$ were measured (Viasys Healthcare Cardiosoft, version 20). Cardiac output can be estimated from $\mathrm{VO}_{2}$ as: $\mathrm{CO}=\frac{100 \times \mathrm{VO}_{2}}{5.721+\left(0.1047 \times \% \mathrm{VO}_{2 \max }\right)}[13]$. 


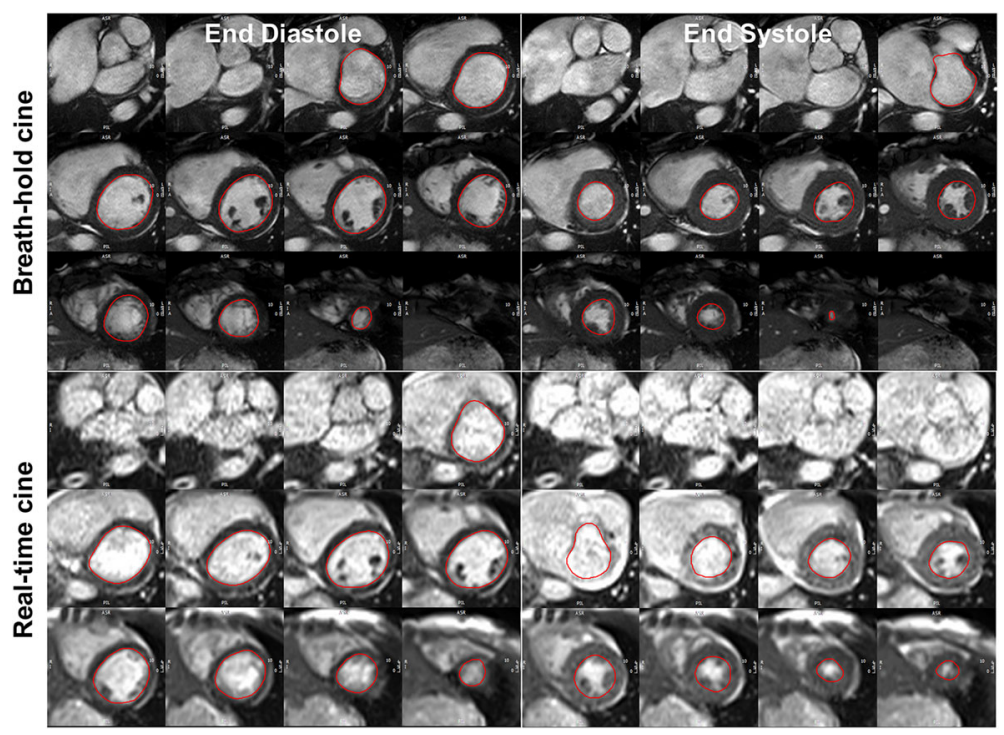

Fig. 3 Endocardial contours for volume measurements of breath-hold cine and real-time cine images

\section{Statistical analysis}

All continuous variables were assessed for normal distribution and presented as mean \pm standard deviation or median [interquartile range (IQR)], as appropriate. Heart rate was normalized to the percent change between rest (0\%) and peak heart rate (100\%).

Pearson's correlation and linear regression were used to assess the association between exercise capacities measured from exCMR and from CPET. Fixed and proportional biases with $95 \%$ limits of agreement between these two techniques were assessed using the Bland-Altman analysis. The performance of exCMR was assessed using the $c$ statistics for discrimination [area under the receiver operating curve].

All statistical analyses were performed using GraphPad Prism 7 (GraphPad Software, Inc., San Diego, CA) and SPSS version 24 (IBM Corp., Armonk, NY). A 2-sided $P$ $<0.05$ was considered statistically significant.

\section{Results}

\section{Baseline characteristics}

Athletes had larger cardiac volumes and increased LV mass compared to healthy individuals $(P<0.05$ for all comparisons). In athletes and healthy individuals, there were no sex-related differences in indexed SV and cardiac index $(\mathrm{CI})$ ( $\mathrm{P}>0.05$ for all comparisons, Table 1). All athletes exercised more than $10 \mathrm{~h}$ a week and considered active based on the questionnaire. On average, healthy volunteers exercised between 1 and 3 $\mathrm{h}$ a week and were considered moderately active based on the questionnaire.

\section{Exercise CMR protocol}

All subjects exercised until they felt they could not continue. Median exercise duration was 7 [IQR: 7-10] minutes in healthy volunteers and 9 [IQR: 8-11] minutes in athletes. The duration of exercise cessation for image acquisition in between each stage ranged between 15 and $25 \mathrm{~s}$, depending on the heart rate. As heart rate increased in the later stages of exercise, the cardiac cycle length became shorter and therefore, shorter acquisition times. The drop in heart rate from the end of exercise to the end of image acquisition was $9 \pm 6 \mathrm{bpm}$ in healthy volunteers and $16 \pm 9 \mathrm{bpm}$ in athletes. The exCMR was well-tolerated by all participants (median satisfaction score of 4 [IQR: 3-4]). At baseline, LV EDV and ESV measured from the baseline real-time and breath-hold cine images demonstrated excellent agreement (Indexed EDV: mean difference of $0.8 \pm 1.8 \mathrm{~mL} / \mathrm{m}^{2}$; Indexed ESV: $0.4 \pm 1.9 \mathrm{~mL} / \mathrm{m}^{2}$ ).

In the intermediate stages of exercise, there were notable differences between athletes and healthy volunteers. In athletes, LV EDV increased at about $75 \%$ peak heart rate and remained elevated at peak exercise despite increasing heart rate; whilst in healthy volunteers, LV EDV peaked earlier (about 50\% peak heart rate) and decreased subsequently (Fig. 4). LV ESV decreased at every stage of exercise in athletes and healthy volunteers (Figs. 4 and 5). This accounted for the different SV profiles: indexed SV peaked at 75\% peak heart rates in both athletes and healthy volunteers. Whilst indexed SV remained elevated at peak exercise in athletes, it decreased in healthy volunteers (Fig. 4). The chronotropic response in the later stages of exercise differed between athletes and less fit 
Table 1 Baseline characteristics and ventricular measurements

\begin{tabular}{|c|c|c|c|}
\hline Parameters at baseline & Healthy volunteers $(n=16)$ & Athletes $(n=11)$ & $P$ value \\
\hline \multicolumn{4}{|l|}{ Clinical variables } \\
\hline Age, years & 26 [25-33] & 29 [28-39] & 0.121 \\
\hline Males, n (\%) & $7(44)$ & $5(45)$ & 0.93 \\
\hline Systolic blood pressure, $\mathrm{mmHg}$ & 117 [107-123] & 119 [107-127] & 0.815 \\
\hline Diastolic blood pressure, $\mathrm{mmHg}$ & 67 [59-75] & $68[66-74]$ & 0.446 \\
\hline Heart rate, beats per minute & 68 [59-74] & $54[48-61]$ & 0.026 \\
\hline Body surface area, $\mathrm{m}^{2}$ & $1.64[1.51-1.96]$ & $1.64[1.50-1.97]$ & 0.394 \\
\hline \multicolumn{4}{|l|}{ Cardiovascular variables } \\
\hline LV mass, $g$ & 76 [65-97] & 100 [93-122] & 0.008 \\
\hline LV end-diastolic volume, mL & $141[115-169]$ & $169[145-188]$ & 0.121 \\
\hline LV end-systolic volume, mL & $54[43-79]$ & 79 [62-83] & 0.05 \\
\hline LV stroke volume, mL & $88[74-104]$ & $101[80-104]$ & 0.422 \\
\hline Cardiac output, L/min & $5.8[4.5-6.3]$ & $5.6[4.0-6.6]$ & 0.610 \\
\hline RV end-diastolic volume, $\mathrm{mL}$ & 150 [121-197] & 189 [154-208] & 0.178 \\
\hline RV end-systolic volume, $\mathrm{mL}$ & 69 [45-99] & $90[74-100]$ & 0.231 \\
\hline Indexed LV mass, $\mathrm{g} / \mathrm{m}^{2}$ & $47[40-53]$ & $66[6-74]$ & $<0.001$ \\
\hline Indexed LV end-diastolic volume, $\mathrm{mL} / \mathrm{m}^{2}$ & $82[75-94]$ & 102 [96-110] & $<0.001$ \\
\hline Indexed LV end-systolic volume, $\mathrm{mL} / \mathrm{m}^{2}$ & $33[28-40]$ & 48 [43-49] & 0.001 \\
\hline Indexed LV stroke volume, $\mathrm{mL} / \mathrm{m}^{2}$ & $52[48-54]$ & 56 [54-63] & 0.003 \\
\hline Cardiac index, $\mathrm{L} / \mathrm{min} / \mathrm{m}^{2}$ & $3.3[2.8-3.9]$ & $3.4[2.6-4.1]$ & 0.865 \\
\hline Indexed RV end-diastolic volume, $\mathrm{mL} / \mathrm{m}^{2}$ & $91[81-107]$ & $112[106-122]$ & 0.002 \\
\hline Indexed RV end-systolic volume, $\mathrm{mL} / \mathrm{m}^{2}$ & $40[30-53]$ & $56[45-59]$ & 0.023 \\
\hline
\end{tabular}

Abbreviations: $L V$ left ventricle, $R V$ right ventricle

healthy volunteers: increased heart rate augment CI in athletes, but not in healthy volunteers (Fig. 4).

At peak exercise, heart rate increased by $238 \pm 39 \%$ and $264 \pm 32 \%$ in healthy volunteers and athletes, respectively. This corresponded to $83 \pm 6 \%$ and $78 \pm 7 \%$ of age-predicted maximal heart rate in healthy individuals and athletes, respectively. Despite similar CI at rest (3.3 [IQR:2.8-3.9] $\mathrm{L} / \mathrm{min} / \mathrm{m}^{2}$ versus 3.4 [IQR: $\left.2.6-4.1\right] \mathrm{L} / \mathrm{min} / \mathrm{m}^{2} ; P=0.87$ ), athletes had increased $\mathrm{CI}$ compared to healthy volunteers at peak exercise (12.2 [IQR: $10.2-13.5] \mathrm{L} / \mathrm{min} / \mathrm{m}^{2}$ versus 8.9 [IQR: $7.5-10.1$ ] $\mathrm{L} / \mathrm{min} / \mathrm{m}^{2} ; P<0.001$; Table 2). Similar to baseline values, there were no sex-related differences in $\mathrm{CI}$ at peak exercise in athletes and healthy volunteers ( $\mathrm{P}>0.05$ for all comparisons). Therefore, we combined both sexes in the subsequent analysis. Unlike at rest, $\mathrm{CI}$ at peak exercise demonstrated excellent ability in differentiating athletes from healthy volunteers (c-statistics $=0.48[95 \%$ confidence interval: $0.23-0.72$ ] versus 0.95 [95\% confidence interval: 0.87 to 1.00 ]; $P<0.0001$ for comparison).

\section{Reproducibility of exCMR and comparison with CPET}

The exCMR protocol demonstrated excellent scanrescan reproducibility with no difference in CI between the two scans performed at least 7 days apart $\left(0.2 \pm 0.5 \mathrm{~L} / \mathrm{min} / \mathrm{m}^{2}\right.$, Fig. 6). Moreover, we observed excellent inter-observer variability in the assessment of cardiac volumes (LVEDV: $2.8 \pm 5.2 \mathrm{~mL}$; LVESV: $-0.5 \pm 5.2 \mathrm{~mL}$ ).

Of the 20 individuals who underwent both exercise tests, $\mathrm{VO}_{2 \max }$ was significantly higher in athletes compared to healthy individuals (50.7 [IQR: 39.4 to 56.9] $\mathrm{mL} / \mathrm{kg} / \mathrm{min}$ versus 29.8 [IQR: 28.3 to 34.0 ] $\mathrm{mL} / \mathrm{kg} / \mathrm{min}$, respectively; $P<0.001)$. Despite similar $\mathrm{CI}$ achieved (1.7 $\pm 1.8 \mathrm{~L} / \mathrm{min} / \mathrm{m}^{2}$; Fig. 7), the maximal workload on the CMR supine ergometer was significantly lower compared to upright cycle CPET (Athletes: 200 [IQR: 175225] W versus 237 [IQR: 213-300] W, $P=0.007$; healthy volunteers: 125 [IQR: 100-150] W versus 188 [IQR: 144-206] W, $P=0.007$ ). All participants exercised to the point they could not continue: the maximal exCMR workload was equivalent to the CPET workload at 80 [IQR: $70-86] \% \mathrm{VO}_{2 \max }$, much higher than the maximal workload defined at $60 \% \mathrm{VO}_{2 \max }$ in other studies $[3,5]$. The CI from exCMR correlated very well with both CPET-derived CI $(r=0.83, P<0.001$; Fig. 7$)$ and CPET $\mathrm{VO}_{2 \max } \quad(r=0.64, \quad P=0.003)$. Compared to $\mathrm{VO}_{2 \max }$, exCMR-derived $\mathrm{CI}$ at peak exercise demonstrated similar ability to differentiate healthy 

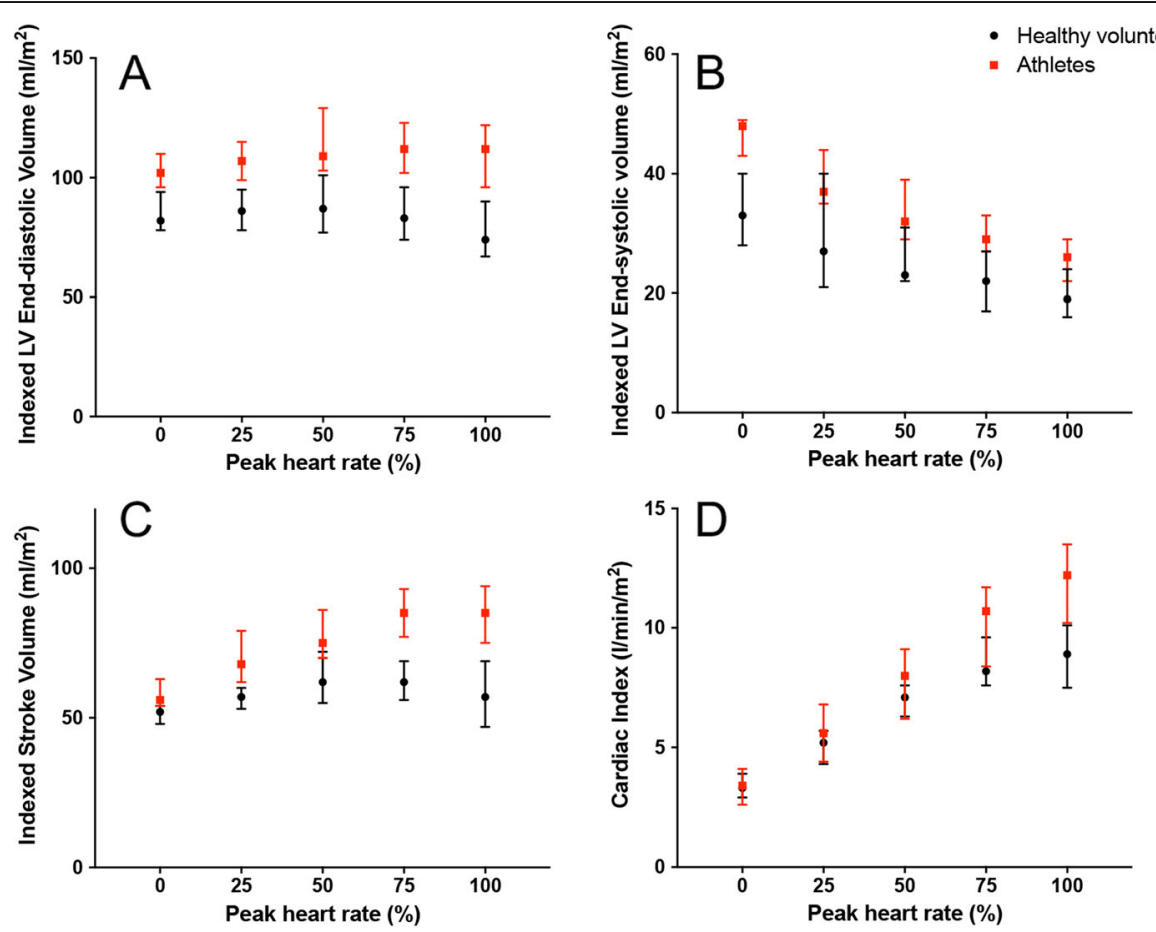

Fig. 4 Exercise Cardiac Reserve in Athletes and Healthy Volunteers. Changes in indexed LV end-diastolic volume (a), indexed LV end-systolic volume (b), indexed stroke volume (c) and cardiac index (d) during exercise in healthy volunteers and athletes. Data presented in median (dots) and interquartile range (bars)

volunteers from athletes (c-statistics $=0.84[95 \%$ confidence interval: $0.62-1.00]$ versus 0.95 [95\% confidence interval: 0.87 to 1.00$]$, respectively; $P=0.292$ for comparison).

\section{Discussion}

We have developed an exCMR protocol with excellent inter-observer and scan-rescan reproducibility. We have also observed excellent correlations and agreement in exercise capacity between exCMR and the gold standard CPET. Using the exercise protocol, we were able to characterise exercise physiology at every stage; and demonstrated excellent ability in differentiating athletes from healthy volunteers (c-statistics $=0.95$ [95\% CI: 0.87 to 1.00]; $P<0.0001$ ).

ExCMR requires appropriate exercise equipment, rapid and robust real-time image acquisition to accommodate free-breathing exercise protocols. A previous study tested the feasibility of treadmill placed outside the MR scanner room and used breath-hold imaging protocol [14]. This took $60-90 \mathrm{~s}$ to complete post-exercise imaging because of the time needed to transfer patients from the treadmill to the MR scanner and image acquisition. Subsequent studies adopted a modified treadmill in the MR scanner room and free-breathing acquisition protocols, with some improvement in post-exercise imaging time $[6-8,15]$. Although the acquisition time was faster compared to previous studies, imaging was only carried out at maximal exercise and not at every stage (which is a potential strength in supine bike protocols).

The recent use of in-scanner cycle ergometer has eliminated any delay in transferring patients into the scanner after exercise, but excessive motion poses a challenge in image acquisition during exercise [5]. Ungated real-time cine imaging and retrospective synchronisation of respiratory cycles have been used to reduce motion, at the expense of increased image acquisition time and complex image post-processing [5]. Although phasecontrast imaging can reduce image acquisition time, it is not able to assess wall motion abnormalities in myocardial ischemia $[4,16,17]$.

We demonstrated rapid acquisition of free-breathing peak-exercise cine images within 13 to $17 \mathrm{~s}$ and superior spatiotemporal resolution (spatial resolution: $3.3 \times 2.3$ $\mathrm{mm}$; temporal resolution $<40 \mathrm{~ms}$ ). In our exercise protocol, we used two different acquisition duration $(1500 \mathrm{~ms}$ and $1200 \mathrm{~ms}$ per slice for heart rates less than and more than 80 , respectively) to ensure at least 2 cardiac cycles that would adequately identify the end diastolic and systolic phases. Importantly, the faster acquisition did not compromise image quality. Moreover, the increased number of coil elements may reduce artefacts that may affect the accurate assessment of cardiac volumes and function; and wall motion abnormalities. Indeed, we 


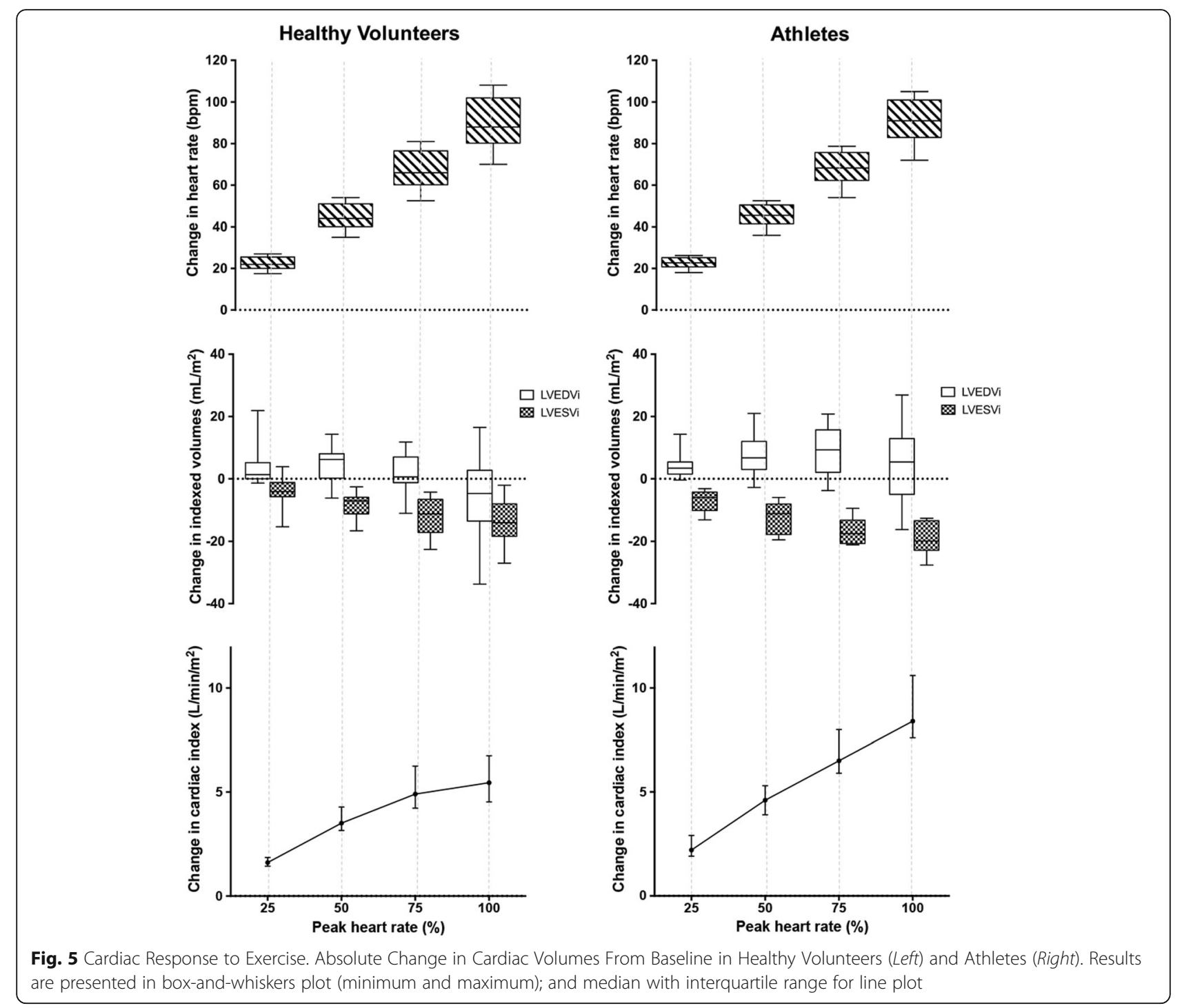

were able to achieve excellent scan-rescan and interobserver variability; and did not observe any difference in ventricular volumes when compared using standard breath-hold and our free-breathing protocols.

CPET is widely accepted as the most reliable and objective test for assessing cardiac reserve in a variety of cardiac conditions, such as heart failure and distinguishing physiologic from pathologic left ventricular hypertrophy $[18,19]$. We used similar exercise protocols for both the in-scanner cycle and upright cycle in CPET. All participants were given clear instructions to exercise until they were not able to continue anymore to ensure maximal exercise capacity was achieved. Of note, the maximum workload on the supine cycle ergometer was lower compared to upright cycle CPET. It is perhaps not surprising that the maximal workload would vary across different exercise stress modalities because of different cardiac responses. Despite different workload attained, supine and upright cycling demonstrated similar $\mathrm{VO}_{2 \max }$ $[20,21]$, but lower than treadmill CPET [11, 22]. Moreover, we observed excellent correlation and agreement in cardiac index between supine exCMR and upright cycle CPET, supporting the validity of the study.

During exCMR, we observed differences in exercise physiology between athletes and healthy individuals. The rate of increase of heart rate in response to exercise was similar in both groups (Fig. 5). Whilst athletes had augmented diastolic filling (increased LV EDV) and improved contractility (decreased LV ESV) throughout the range of exercise intensity tested, healthy volunteers attained peak diastolic filling and contractility earlier (at about $50 \%$ peak heart rate). At higher exercise intensity, increased chronotropic response further augment cardiac output in athletes but not in the less fit healthy volunteers. In healthy volunteers, the rapid heart rates reduced diastolic filling and therefore, stroke volumes at 
Table 2 Peak exercise comparison between healthy volunteers and athletes

\begin{tabular}{|c|c|c|c|}
\hline Parameters at peak exercise & Healthy volunteers $(n=16)$ & Athletes $(n=11)$ & $P$ value \\
\hline \multicolumn{4}{|l|}{ Clinical variables } \\
\hline Heart rate, bpm & 156 [150-165] & 145 [135-158] & 0.071 \\
\hline Systolic blood pressure, $\mathrm{mmHg}$ & 128 [103-192] & 150 [120-165] & 0.640 \\
\hline Diastolic blood pressure, $\mathrm{mmHg}$ & 90 [59-134] & 68 [40-109] & 0.379 \\
\hline Maximum exercise power, W & 125 [107-150] & $200[175-225]$ & 0.011 \\
\hline \multicolumn{4}{|l|}{ Cardiovascular variables } \\
\hline LV end-diastolic volume, mL & 135 [104-152] & $171[150-208]$ & 0.005 \\
\hline LV end-systolic volume, $\mathrm{mL}$ & $32[26-47]$ & $42[36-46]$ & 0.178 \\
\hline LV stroke volume, $\mathrm{mL}$ & 96 [73-121] & $129[115-158]$ & 0.003 \\
\hline Cardiac output, L/min & 15 [12-19] & 20 [16-23] & 0.023 \\
\hline Indexed LV end-diastolic volume, $\mathrm{ml} / \mathrm{m}^{2}$ & 75 [67-90] & $112[96-122]$ & $<0.001$ \\
\hline Indexed LV end-systolic volume, $\mathrm{ml} / \mathrm{m}^{2}$ & 20 [16-24] & 26 [22-28] & 0.044 \\
\hline Indexed stroke volume, $\mathrm{ml} / \mathrm{m}^{2}$ & 57 [47-69] & 85 [75-94] & $<0.001$ \\
\hline Cardiac index, $\mathrm{L} / \mathrm{min} / \mathrm{m}^{2}$ & $8.9[7.5-10.1]$ & $12.2[10.2-13.5]$ & $<0.001$ \\
\hline
\end{tabular}

Abbreviations: $L V$ left ventricle, $R V$ right ventricle

peak exercise. There are some uncertainties in the mechanisms associated with cardiac output augmentation in athletes and non-athletes [23-27]. Our study adds novelty by demonstrating mechanistic differences in exercise profiles between athletes and healthy volunteers using the same exercise protocol.

\section{Clinical implications}

The study highlighted the potential of further extending the clinical applications of CMR to assess cardiac reserve. In addition to CMR being the gold standard for assessing left ventricular mass and cardiac volumes, it is the only imaging modality that can detect myocardial fibrosis non-invasively. The combination of these techniques in a single imaging modality offers valuable diagnostic insights in individuals with cardiac pathologies, which not achievable with CPET. The ability to assess cardiac physiology and function at every stage of exercise provides a unique opportunity to characterise and differentiate the exercise profiles between individuals. In this study, we have demonstrated one such potential application: peak exercise CI significantly outperformed $\mathrm{CI}$ at rest and had similar performance as $\mathrm{VO}_{2 \max }$ in differentiating athletes from healthy volunteers. This technique holds promise in distinguishing physiologic from pathologic myocardial biology in patients with LV dilatation, hypertrophy or mildly impaired systolic ejection fraction [28, 29]. The excellent reproducibility adds strength to use exCMR for serial assessments.

\section{Limitations}

The CMR protocol required a brief period of stopping exercise at the end of every stage in order to minimise excessive motion and ECG artefacts during image acquisition. This resulted in a small drop in heart rate $(9 \pm 6$ $\mathrm{bpm}$ in controls and $16 \pm 9 \mathrm{bpm}$ in athletes). However, the effect may be less significant in patients with cardiac pathologies or individuals who are older and less
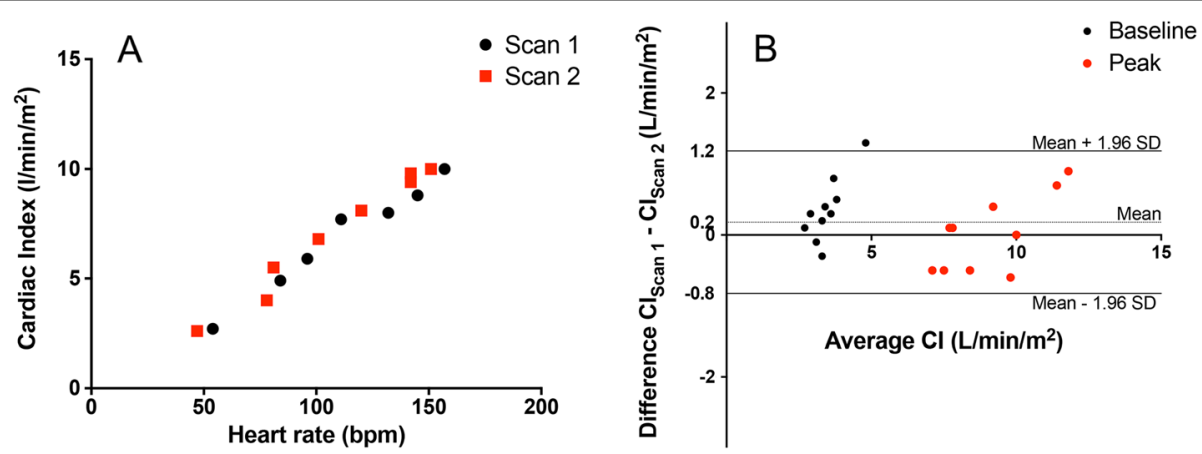

Fig. 6 Scan-rescan reproducibility. Example of the exercise profile of an individual performed in the two scans (a); Bland-Altman plot of the difference in cardiac index measured between the two scans (b) 

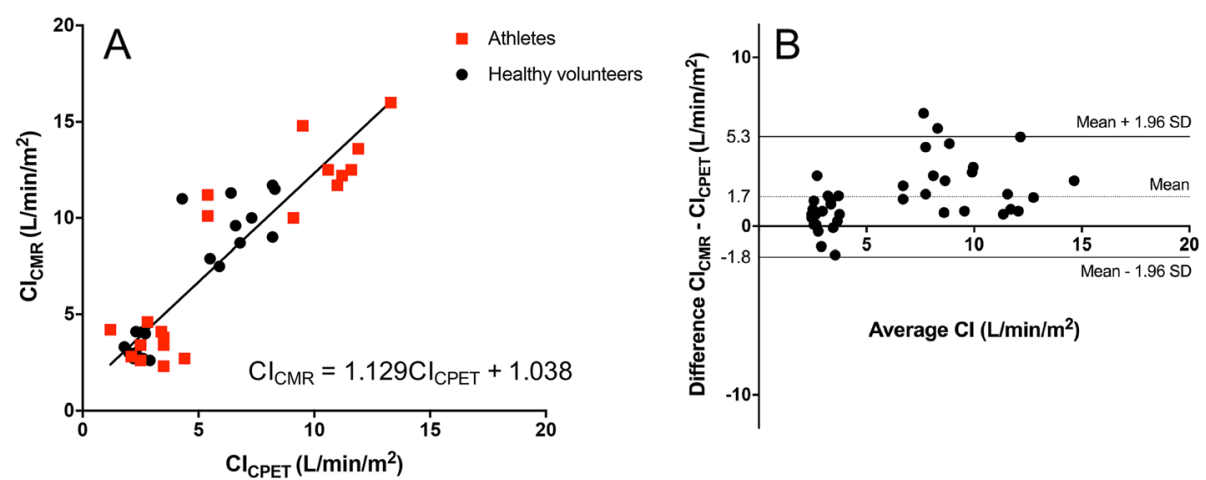

Fig. 7 Correlation and Agreement in Cardiac Index Derived from Exercise CMR and CPET. Linear regression of cardiac index values measured from CMR and derived from CPET at rest and peak exercise (a) and Bland-Altman plot of the difference between cardiac index measured from CMR and estimated from CPET, at rest and peak exercise (b)

physically-active. The maximal heart rate achieved using this exCMR protocol was less than the recommended $85 \%$ age-predicted maximal heart rate (APMHR) commonly used in defining an adequate treadmill stress test $[11,30]$. This may affect the diagnostic accuracy in assessing myocardial ischemia. However, it is well described that heart rates achieved with supine cycling is lower than exercise treadmill [22, 31, 32]; and previous echo studies have demonstrated similar diagnostic accuracies between supine and treadmill exercise stress, despite a large proportion of patients not achieving the $85 \%$ APMHR [31-33]. It is conceivable that supine exercise stress may not be suitable in some individuals (particularly those who are not accustomed to cycling) because of the postural effects on musculoskeletal fatigue.

\section{Conclusions}

These data demonstrate the feasibility, accuracy and reproducibility of an in-scanner exercise protocol using a CMR-compatible cycle ergometer. Future studies will examine its clinical utility in a variety of cardiac pathologies.

\section{Additional files}

Additional file 1: Video S1. Breath-hold cine images at baseline. (MOV $857 \mathrm{~KB}$ )

Additional file 2: Video S2. Real-time cine images at baseline. (MOV $1010 \mathrm{~KB}$ )

Additional file 3: Video S3. Real-time cine images at peak exercise. (MOV 780 KB)

\section{Abbreviations}

APMHR: Age-predicted maximal heart rate; Cl: Cardiac Index; CMR: Cardiovascular magnetic resonance; CO: Cardiac output; CPET: Cardiopulmonary exercise test; EDV: End-diastolic volume; ESV: End-systolic volume; exCMR: Exercise CMR; LV: Left ventricular/left ventricle; SV: Stroke volume; TGRAPPA: Temporal generalized auto-calibrating partially parallel acquisition; $\mathrm{VO}_{2}$ : Oxygen consumption; $\mathrm{VO}_{2 \text { max }}$ : Oxygen consumption at peak exercise

\section{Acknowledgements}

The authors would like to thank the physiotherapists of the Changi Sports Medicine Centre, Changi General Hospital and the radiographers of the National Heart Centre Singapore for conducting the tests and Mr Chan Cheow Hiong and Mrs Agnes Low for their financial donation to the study.

\section{Funding}

Duke-NUS Goh Cardiovascular Research Award, National Medical Research Council Transition Award (NMRC/TA/0034/2015), Philanthropy Fund (Mr Chan Cheow Hiong and Mrs Agnes Low).

\section{Availability of data and materials}

The datasets during and/or analysed during the current study available from the corresponding author on reasonable request.

\section{Authors' contributions}

$T T L$ conceived the study design, analysed the images, interpreted the data and drafted the manuscript. JAB optimized imaging sequence, acquired the scans and analysed the images and contributed to the intellectual content of the manuscript. AET acquired the scans for all the patients in the study, involved in the acquisition of data and contributed to the intellectual content of the manuscript. PYH involved in the acquisition of data and contributed to the intellectual content of the manuscript. BS analysed the data, developed in-house add-in program for analysis and contributed to the intellectual content of the manuscript. RCCT involved in the design of cardiopulmonary test, acquired the data and contributed to the intellectual content of the manuscript. JSJG optimized imaging sequence and contributed to the intellectual content of the manuscript. YCC optimized imaging sequence and contributed to the intellectual content of the manuscript. DOR interpreted the data and involved in the critical revision of the manuscript. SC conceived the study design, interpreted the data and involved in the critical revision of the manuscript. CCWL conceived the study design, interpreted the data and drafted the manuscript. All authors read and approved the final manuscript.

\section{Competing interests}

The authors declare that they have no competing interests.

\section{Consent for publication}

Not applicable.

\section{Ethics approval and consent to participate}

written informed consent was obtained from all participants. The study protocol was approved by Singhealth Centralised Institutional Review Board, reference number 2016/2256 and 2016/2050.

\section{Author details}

${ }^{1}$ National Heart Centre Singapore, 5 Hospital Drive, Singapore 169609, Singapore. ${ }^{2}$ Changi General Hospital, Singapore, Singapore. ${ }^{3}$ Siemens Healthineers, Erlangen, Germany. ${ }^{4}$ MRC London Institute of Medical Sciences, 
London, UK. ${ }^{5}$ Duke-NUS Medical School, Singapore, Singapore. ${ }^{6}$ National Heart and Lung Institute, Imperial College, London, UK.

Received: 14 December 2016 Accepted: 6 January 2017 Published online: 23 January 2017

\section{References}

1. Sechtem U, Pflugfelder PW, Gould RG, Cassidy MM, Higgins CB. Measurement of right and left ventricular volumes in healthy individuals with cine MR imaging. Radiology. 1987;163(3):697-702.

2. Grothues F, Smith GC, Moon JC, Bellenger NG, Collins P, Klein HU, Pennell DJ. Comparison of interstudy reproducibility of cardiovascular magnetic resonance with two-dimensional echocardiography in normal subjects and in patients with heart failure or left ventricular hypertrophy. Am J Cardiol. 2002;90(1):29-34.

3. Roest AA, Kunz P, Lamb HJ, Helbing WA, van der Wall EE, de Roos A. Biventricular response to supine physical exercise in young adults assessed with ultrafast magnetic resonance imaging. Am J Cardiol. 2001;87(5):601-5.

4. Pieles GE, Szantho G, Rodrigues JC, Lawton CB, Stuart AG, Bucciarelli-Ducci C, Turner MS, Williams CA, Tulloh RM, Hamilton MC. Adaptations of aortic and pulmonary artery flow parameters measured by phase-contrast magnetic resonance angiography during supine aerobic exercise. Eur J Appl Physiol. 2014;114(5):1013-23

5. La Gerche A, Claessen G, Van de Bruaene A, Pattyn N, Van Cleemput J, Gewillig M, Bogaert J, Dymarkowski S, Claus P, Heidbuchel H. Cardiac MRI: a new gold standard for ventricular volume quantification during highintensity exercise. Circ Cardiovasc Imaging. 2013;6(2):329-38.

6. Jekic M, Foster EL, Ballinger MR, Raman SV, Simonetti OP. Cardiac function and myocardial perfusion immediately following maximal treadmill exercise inside the MRI room. J Cardiovasc Magn Reson. 2008;10.

7. Raman SV, Dickerson JA, Jekic M, Foster EL, Pennell ML, McCarthy B, Simonetti OP. Real-time cine and myocardial perfusion with treadmill exercise stress cardiovascular magnetic resonance in patients referred for stress SPECT. J Cardiovasc Magn Reson. 2010;12(1):1-9.

8. Thavendiranathan P, Dickerson JA, Scandling D, Balasubramanian V, Pennell ML, Hinton A, Raman SV, Simonetti OP. Comparison of treadmill exercise stress cardiac MRI to stress echocardiography in healthy volunteers for adequacy of left ventricular endocardial wall visualization: A pilot study. J Magn Reson Imaging. 2014;39(5):1146-52.

9. Raman SV, Dickerson JA, Mazur W, Wong TC, E BS, Min JK, Scandling D, Bartone C, Craft JT, Thavendiranathan P, et al. Diagnostic Performance of Treadmill Exercise Cardiac Magnetic Resonance: The Prospective, Multicenter Exercise CMR's Accuracy for Cardiovascular Stress Testing (EXACT) Trial. J Am Heart Assoc. 2016:5(8):e003811.

10. Lauer MS, Francis GS, Okin PM, Pashkow FJ, Snader CE, Marwick TH. Impaired chronotropic response to exercise stress testing as a predictor of mortality. JAMA. 1999;281(6):524-9.

11. Fletcher GF, Balady GJ, Amsterdam EA, Chaitman B, Eckel R, Fleg J, Froelicher VF, Leon AS, Pina IL, Rodney R, et al. Exercise standards for testing and training: a statement for healthcare professionals from the American Heart Association. Circulation. 2001;104(14):1694-740.

12. Khaw KT, Jakes $R$, Bingham S, Welch A, Luben R, Day N, Wareham N. Work and leisure time physical activity assessed using a simple, pragmatic, validated questionnaire and incident cardiovascular disease and all-cause mortality in men and women: The European Prospective Investigation into Cancer in Norfolk prospective population study. Int J Epidemiol. 2006;35(4):1034-43.

13. Stringer WW, Hansen JE, Wasserman K. Cardiac output estimated noninvasively from oxygen uptake during exercise. J Appl Physiol. 1997;82(3):908-12.

14. Rerkpattanapipat P, Gandhi SK, Darty SN, Williams RT, Davis AD, Mazur W, Clark HP, Little WC, Link KM, Hamilton CA, et al. Feasibility to detect severe coronary artery stenoses with upright treadmill exercise magnetic resonance imaging. Am J Cardiol. 2003;92.

15. Lafountain RA, da Silveira JS, Varghese J, Mihai G, Scandling D, Craft J, Swain CB, Franco V, Raman SV, Devor ST, et al. Cardiopulmonary exercise testing in the MRI environment. Physiol Meas. 2016;37(4):N11-25.

16. Barber NJ, Ako EO, Kowalik GT, Steeden JA, Pandya B, Muthurangu V. MR augmented cardiopulmonary exercise testing-a novel approach to assessing cardiovascular function. Physiol Meas. 2015:36(5):N85-94.

17. Mohiaddin RH, Gatehouse PD, Firmin DN. Exercise-related changes in aortic flow measured with spiral echo-planar MR velocity mapping. J Magn Reson Imaging. 1995;5(2):159-63.
18. Guazzi M, Arena R, Halle M, Piepoli MF, Myers J, Lavie CJ. 2016 Focused Update: Clinical Recommendations for Cardiopulmonary Exercise Testing Data Assessment in Specific Patient Populations. Circulation. 2016;133(24): e694-711.

19. Sharma S, Elliott PM, Whyte G, Mahon N, Virdee MS, Mist B, McKenna WJ. Utility of metabolic exercise testing in distinguishing hypertrophic cardiomyopathy from physiologic left ventricular hypertrophy in athletes. J Am Coll Cardiol. 2000;36(3):864-70.

20. Leyk D, Essfeld D, Hoffmann U, Wunderlich HG, Baum K, Stegemann J. Postural effect on cardiac output, oxygen uptake and lactate during cycle exercise of varying intensity. Eur J Appl Physiol Occup Physiol. 1994;68(1): 30-5.

21. Quinn TJ, Smith SW, Vroman NB, Kertzer R, Olney WB. Physiologic responses of cardiac patients to supine, recumbent, and upright cycle ergometry. Arch Phys Med Rehabil. 1995:76(3):257-61.

22. Proctor DN, Sinning WE, Bredle DL, Joyner MJ. Cardiovascular and peak VO2 responses to supine exercise: effects of age and training status. Med Sci Sports Exerc. 1996;28(7):892-9.

23. Holverda S, Gan CT, Marcus JT, Postmus PE, Boonstra A, Vonk-Noordegraaf A. Impaired stroke volume response to exercise in pulmonary arterial hypertension. J Am Coll Cardiol. 2006;47(8):1732-3.

24. Crawford MH, Petru MA, Rabinowitz C. Effect of isotonic exercise training on left ventricular volume during upright exercise. Circulation. 1985;72(6):1237-43.

25. Ginzton LE, Conant R, Brizendine M, Laks MM. Effect of long-term high intensity aerobic training on left ventricular volume during maximal upright exercise. J Am Coll Cardiol. 1989;14(2):364-71.

26. Rowland T. Echocardiography and circulatory response to progressive endurance exercise. Sports Med. 2008;38(7):541-51.

27. Gledhill N, Cox D, Jamnik R. Endurance athletes' stroke volume does not plateau: major advantage is diastolic function. Med Sci Sports Exerc. 1994; 26(9):1116-21.

28. Sharma S, Elliott P, Whyte G, Jones S, Mahon N, Whipp B, McKenna WJ. Utility of cardiopulmonary exercise in the assessment of clinical determinants of functional capacity in hypertrophic cardiomyopathy. Am J Cardiol. 2000; 86(2):162-8

29. La Gerche A, Baggish AL, Knuuti J, Prior DL, Sharma S, Heidbuchel H, Thompson PD. Cardiac imaging and stress testing asymptomatic athletes to identify those at risk of sudden cardiac death. JACC Cardiovasc Imaging. 2013;6(9):993-1007.

30. Gibbons RJ, Balady GJ, Beasley JW, Bricker JT, Duvernoy WF, Froelicher VF, Mark DB, Marwick TH, McCallister BD, Thompson Jr PD, et al. ACC/AHA Guidelines for Exercise Testing. A report of the American College of Cardiology/American Heart Association Task Force on Practice Guidelines (Committee on Exercise Testing). J Am Coll Cardiol. 1997:30(1):260-311.

31. Badruddin SM, Ahmad A, Mickelson J, Abukhalil J, Winters WL, Nagueh SF, Zoghbi WA. Supine bicycle versus post-treadmill exercise echocardiography in the detection of myocardial ischemia: a randomized single-blind crossover trial. J Am Coll Cardiol. 1999:33(6):1485-90.

32. Modesto KM, Rainbird A, Klarich KW, Mahoney DW, Chandrasekaran K, Pellikka PA. Comparison of supine bicycle exercise and treadmill exercise Doppler echocardiography in evaluation of patients with coronary artery disease. Am J Cardiol. 2003;91(10):1245-8.

33. Park TH, Tayan N, Takeda K, Jeon HK, Quinones MA, Zoghbi WA. Supine bicycle echocardiography improved diagnostic accuracy and physiologic assessment of coronary artery disease with the incorporation of intermediate stages of exercise. J Am Coll Cardiol. 2007;50(19):1857-63.

\section{Submit your next manuscript to BioMed Central} and we will help you at every step:

- We accept pre-submission inquiries

- Our selector tool helps you to find the most relevant journal

- We provide round the clock customer support

- Convenient online submission

- Thorough peer review

- Inclusion in PubMed and all major indexing services

- Maximum visibility for your research

Submit your manuscript at www.biomedcentral.com/submit 